\title{
UAV PHOTOGRAMMETRY IN REMOTE AREAS - 3D MODELING OF DRAPHAM DZONG BHUTAN
}

\author{
Armin Gruen $^{\mathrm{a},}$, Zhichao Zhang ${ }^{\mathrm{a} *}$, Henri Eisenbeiss ${ }^{\mathrm{b}}$ \\ ${ }^{a}$ Institute of Historic Building Research and Conservation, ETH Zurich, CH-8093 Zurich, Switzerland \\ E-mail: agruen@geod.baug.ethz.ch \\ E-mail: zhichao.zhang@arch.ethz.ch* \\ ${ }^{\mathrm{b}}$ Institute of Geodesy and Photogrammetry, ETH Zurich, CH-8093 Zurich, Switzerland \\ E-mail: henri.eisenbeiss@geod.baug.ethz.ch
}

Theme or Special Session: ICWG I/V

KEY WORDS: Archaeology excavation, UAV Photogrammetry, 3D Modeling,

\begin{abstract}
:
The Buddhist fortress Drapham Dzong is located in the Bumthang District in central Bhutan on a mountain ridge at approximately $2930 \mathrm{~m}$ altitude. A surveying mission was carried out with the goal to produce a textured 3D model of the site, together with a conventional 2D map and an orthophoto. The remote and exposed location of the site makes traditional surveying, including terrestrial laser-scanning a tedious undertaking. Aerial images of reasonable quality are not available in Bhutan. Satellite images, even of very high resolution (50cm footprint) do not show sufficient detail for the modelling of the man-made structures. Therefore this project leads itself very well to the use of an appropriate UAV. This paper describes briefly the raw data acquisition by UAV and terrestrial images, and then focuses on the procedures for data processing. The 3D model of the man-made objects is embedded into a large area model of the environment, generated from a GeoEye-1 stereo model.

Overall, the project is a typical example of a multi-image concept, making use of imagery of quite different resolutions (satellite, UAV, terrestrial). Given a textured accurate 3D model, 2D maps and orthoimages are nothing but derivatives of this general product. The project also shows how lightweight equipment can be used in remote and not easily accessible areas for surveying and 3D modelling purposes.
\end{abstract}
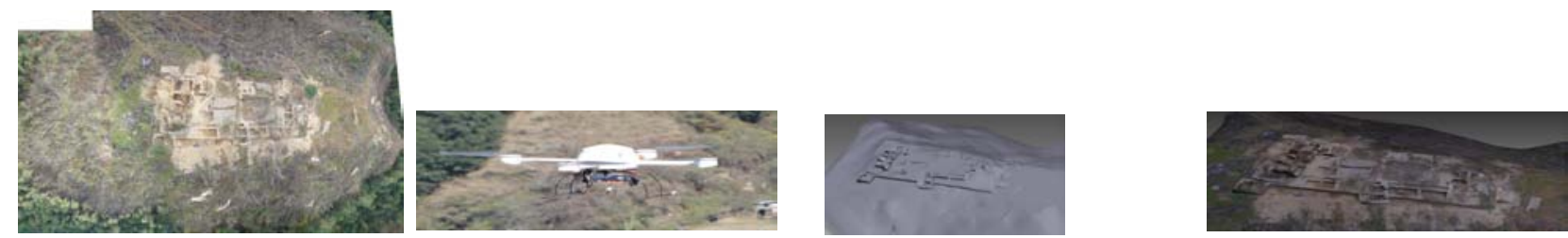

Figure 1: overview of surveying with a UAV system (Microdrone MD4-200) and photogrammetric results achieved from the acquired images: digital surface model, and textured model.

\section{INTRODUCTION}

3D documentation is proven to be an important aspect of archaeology excavation. Though there are many way to do this job, such as laser scanning, in practical condition, the platform and sensor must been chosen to suit the current condition.

Taking into account the considerable size of both the hilltop area (approximately $6500 \mathrm{~m}$ ), it becomes obvious that surveying and mapping of the whole architectural complex cannot be conducted efficiently by means of conventional terrestrial surveying techniques.

Moreover, in the course of the so-called Spatial Turn (the (re)discovery of the importance of space and spatiality in the field of social sciences in the 1980 landscape has turned out to be a considerable object of investigation in modern archaeology (David and Thomas 2008). On the one hand, meticulous hand drawings and measurements of construction details give information about construction techniques, handcrafts, and the building history (see Meyer 2009). On the other hand, the acquisition of the whole architectural complex, including its topographical environment, will shed light on its original social function. A detailed digital three-dimensional mapping of the topography and the remaining structures as discussed here enables the intended analysis of potential cultural acts in which Drapham Dzong once had its function. With a large and detailed landscape model for example, on the basis of the so-

* Corresponding author. 
called Least-Cost-Analysis (Tobler 1993, Fux et al. 2009) potential mule track routes between Assam in the south and Tibet in the north can then be detected.

Thus, a surveying expedition to Drapham Dzong was planned by our multidisciplinary team. The goal of our expedition in November 2009 was the generation of the pre requisites to produce a 3D model of the excavation site and its immediate surroundings. For this purpose our main surveying device was a model helicopter (to be precise a quadrocopter) for the acquisition of very large scale aerial images of the site. These images are the basis for photogrammetric measurements and 3D modeling.

Generally speaking, photogrammetry is an indirect measurement technique which derives from images of an object the object itself with its location, shape and size. The images have to be taken in such a manner from different viewpoints that they overlap each other sufficiently well in their object coverage. The images can then be processed on the basis of known camera and lens parameters. By means of the definition of specific object points visible in several images taken from different points of view, the object's geometry can subsequently be calculated by the use of special photogrammetry software. In the following, the fieldworks conducted in autumn 2009 at Drapham Dzong as well as the ongoing photogrammetric data processing for the purpose of 3D modeling are discussed.

\section{DATA ACQUISITION}

\subsection{Multi-resolution Images}

The high resolution stereo model of the GeoEye satellite, used for modeling the terrain around Drapham Dzong, was donated by the German Aerospace Center Germany (DLR).

Taking into account the considerable size of the object under investigation as well as the complex topography, the modeling with the help of aerial images is most efficient. However, since standard aerial images were not available for this area we had to use high resolution satellite images for the modeling of the wider environment and aerial images taken from a UAV (quadrocopter) for the excavation site modeling. One has to cope with the following factors: The UAV had to be able to carry a digital still video camera at an altitude of more than $3000 \mathrm{~m}$ a.s.l. and the equipment had to be easily transportable from Switzerland to Bhutan and carried to the archaeologists' camp far away from the dirt road.

Considering these factors we opted for a special kind of miniUAV (Unmanned Aerial Vehicle), a so-called quadrocopter, i.e. a UAV with four rotors.

Quadrocopters are usually light weight systems, which normally have a maximum take-off weight of up to $5 \mathrm{~kg}$. These systems have a size of $0.5-2 \mathrm{~m}$ and they are powered by four electro-motors. Several commercial systems are available on the market. Due to the weight limitations, these systems are highly dependent on wind conditions. Moreover, most of the systems can only fly in the manual or assisted flight modes. In the last years, some systems were upgraded by a flight control system, allowing an autonomous flight with a predefined flight path (Eisenbeiss 2009).

For our field campaign at Drapham Dzong the quadrocopter UAV md4-200 of the company microdrones (microdrones 2010) was chosen, since a previous flight test at the Jungfraujoch (Switzerland) at 3,470 m a.s.l. in autumn 2009 had shown that the system is appropriate for flights at this altitude. The system is equipped with a GPS receiver for self-positioning. The camera we selected was a Panasonic Lumix FX35 with a zoom lens.

For the terrestrial image acquisition, such as for the texture of the vertical walls and the documentation of the field work, Nikon D2Xs and D3X, and a Sony DSC-HX1 still video cameras were used.

\subsection{Ground control points}

For the measurement of ground control points (GCPs), we selected two GNSS (Global Navigation Satellite System) receivers GPS 500 (Leica-Geosystems 2010). One of them was used as a reference station and the second in a mobile mode for the individual measurements of the GCPs. The distribution of control points

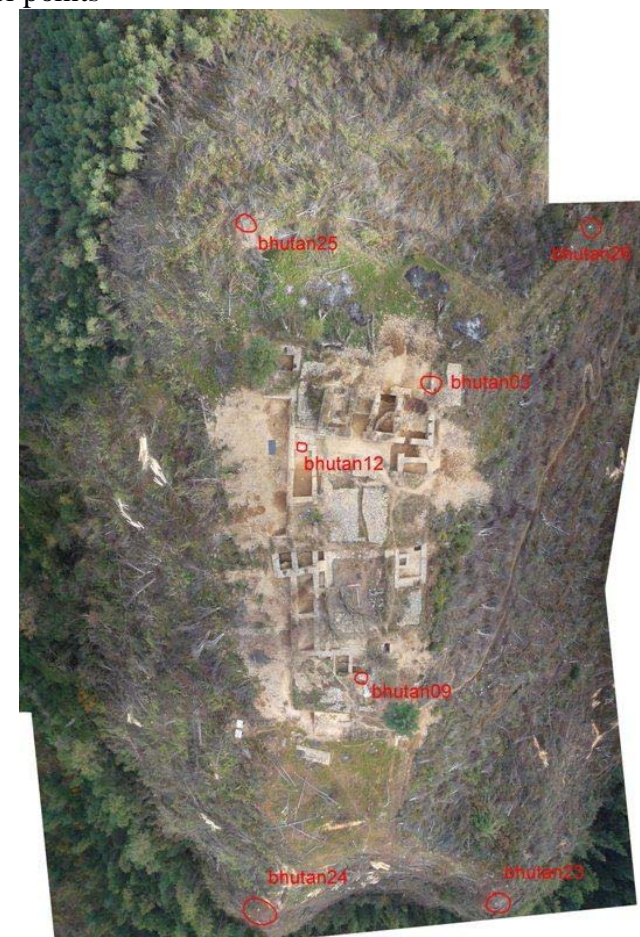

Figure 2: The distribution of ground control points on site.

\section{MULTI-RESOLUTION MODEL GENERATION AND INTEGRATION}

\subsection{Generation of a larger area DSM and ortho-image from GeoEye-1 satellite imagery}

In order to visualize the larger topographic context of Drapham Dzong, a Digital Surface Model (DSM) covering an area of 10.710 .7 square kilometres was generated using two GeoEye1 stereo images with $0.50 \mathrm{~cm}$ ground pixel size (GSD) in PAN mode. The orientation of these two images was already given by Rational Polynomial Coefficients (RPC) which were provided with the images by the German Aerospace Center (DLR). For the purpose of automated DSM generation, we used the SAT-PP software which was developed at the group of Photogrammetry and Remote Sensing at ETH Zurich. The image matching algorithm and its implementation is described in detail in the scientific literature (Zhang 2005).

The software is designed to measure identical points in overlapping images acquired from different positions by means of a complex algorithm that matches interest and grid points 
and also edges. Furthermore, geometrical constraints are applied in order to increase the reliability and accuracy of the measurements. The result is a dense dataset of 3D points and edges which, finally, are combined into a raster dataset that represents the shape of the terrain covered by the stereo scenes. The underlying algorithm has proved to be one of the most accurate automatic methods for DSM generation worldwide. After the DSM was generated, it was used for the generation of an ortho-image using the PAN channel with $0.50 \mathrm{~m}$ ground resolution and the red, green and blue channels $(1.61 \mathrm{~m}$ resolution) of the GeoEye-1 imagery. The resulting orthorectified channels were then combined into a RGB image by means of pan-sharpening using the Brovey-method. For the purpose of visualization, the pan-sharpened ortho-image has been draped over the DSM in order that a textured 3D model of the landscape can be virtually viewed and navigated through (Fig. 3).

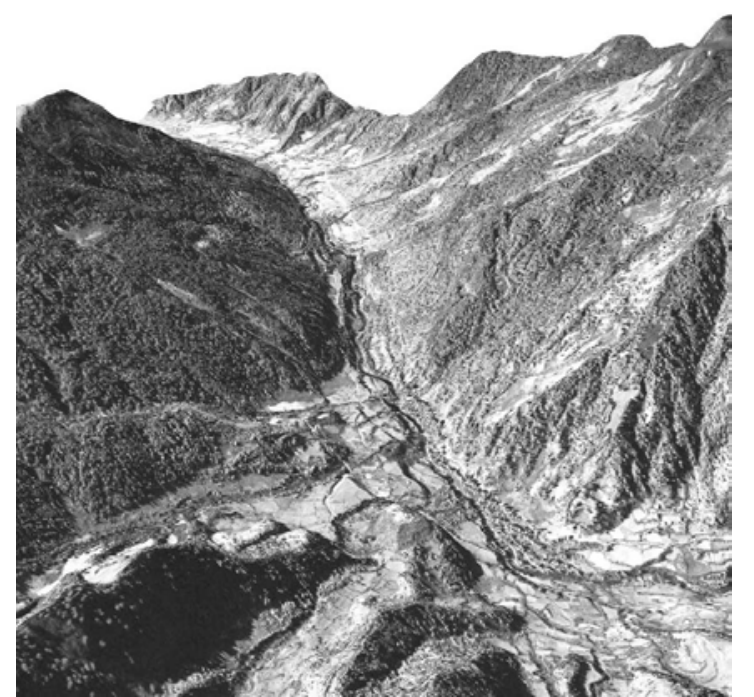

Figure 3: Map showing the hilltop complex and the lower building remains at the foot of the hill. The ruins were photogrammetrically mapped from UAV images, the Digital Surface Model and the contour lines were generated from GeoEye-1 satellite imagery using the software SAT-PP.

\subsection{Geometric Modeling from UAV images}

The data processing of the UAV images consists of the following steps:

1. Calibration of the UAV camera;

2. Image enhancement. Improvement of the radiometric quality of the UAV images;

3. Accurate orientation (georeferencing) of the images;

4. Generation of a Digital Terrain Model (DTM);

5. Measurement and modeling of the man-made (excavated) objects (primarily walls, floors, stairs, etc.);

6. Mapping of image texture onto the DTM and the man-made objects.

The camera calibration, which can be done either in the office or in the field, gives us the exact values of the camera's geometric parameters like camera constant, the coordinates of the principal point, and additional parameters for the correction of the systematic errors introduced by its lens system. Using the criteria image sharpness, orientation, and overlapping areas, we selected a subset for further processing from the whole set of images. This enabled us to obtain two image strips consisting of 8 and 10 images respectively which cover the whole hill, including Drapham Dzong's main building structures. Using the Adobe Photoshop software, the images were enhanced in a manner that effects like over exposure were reduced, and both image contrast and brightness were improved.

After image selection and editing, the UAV images were further processed using the Leica Photogrammetry Suite software (LPS; Leica-Geosystems 2010). For georeferencing we measured in both image strips equally distributed tie points (i.e. significant and static features recognizable in overlapping areas of two or more images) in order to connect (to "tie") the individual images to each other computationally. Only a few tie points were measured manually which could then be used as initial data for the automated tie point generation. The accurate georeferencing of the images, also called "exterior orientation", is a prerequisite for the generation of final products like DTMs, ortho-images or photorealistic 3D models. For this georeferencing, GCPs (ground control points) are also needed. They give information as to how the images (and finally the whole models) are located in an external world coordinate system. In our case, this can either be B\$urofficial surveying/mapping system or the worldwide UTM system. We selected UTM Zone 46 North. Then, stereoscopic measurements of the DTM and the building walls were conducted. This procedure was accomplished using LPS and PhotoModeler (Photomodeler 2010). As additional tools, a stereo screen and stereo glasses were required. Finally, the DTM and the top of the measured walls were textured using the UAV images and our own software. Extra-terrestrial images were acquired during our field campaign for the texturing of the vertical walls.

\subsection{Texture Mapping of UAV Generated Model}

To derive a photo-realistic model of the site, texture mapping is a necessary step. The terrestrial image is for the wall of the model generated from UAV images. a direction based division is adopted to simplify the procedure. The detailed procedure is that separate the model into vertical model and horizontal model. This is more suitable for the procedure of the job, See figure 5. Because we did not orient the terrestrial images, the texture mapping for the vertical wall is not that strictly precise. To get a better result, we use a rubber-sheeting approach to do the texture mapping. A proper number of corresponding points are manually assigned between the images and model. Then this problem can be regarded as a nearly plane parameterization problem. In this simple situation, a 2D Delaunay triangulation is used to parameterize the nearly planar mesh. Based on the control points, an incremental Delaunay construction method is carried out. First of all, three points on the mesh and corresponding images are picked by hand. The picked three points can represent a plane which is nearly parallel to the mesh.

This step is quite important for the texture mapping is mainly based on this plane. So pick the plane as parallel as the nearly planar mesh. Secondly, depending on the dense control points acquired in the last step, the image can be directly mapped onto the nearly planar mesh. However, when the matching step cannot give a good distribution of the control points, a user interface is also supplied to get the corresponding points manually. The manual work is inevitable because of the need of the precise control of the boundary area. After the simple parameterization, the UV coordinate of the vertex on the mesh can be interpolated in the local triangles generated from the 
control points(Fig 4).
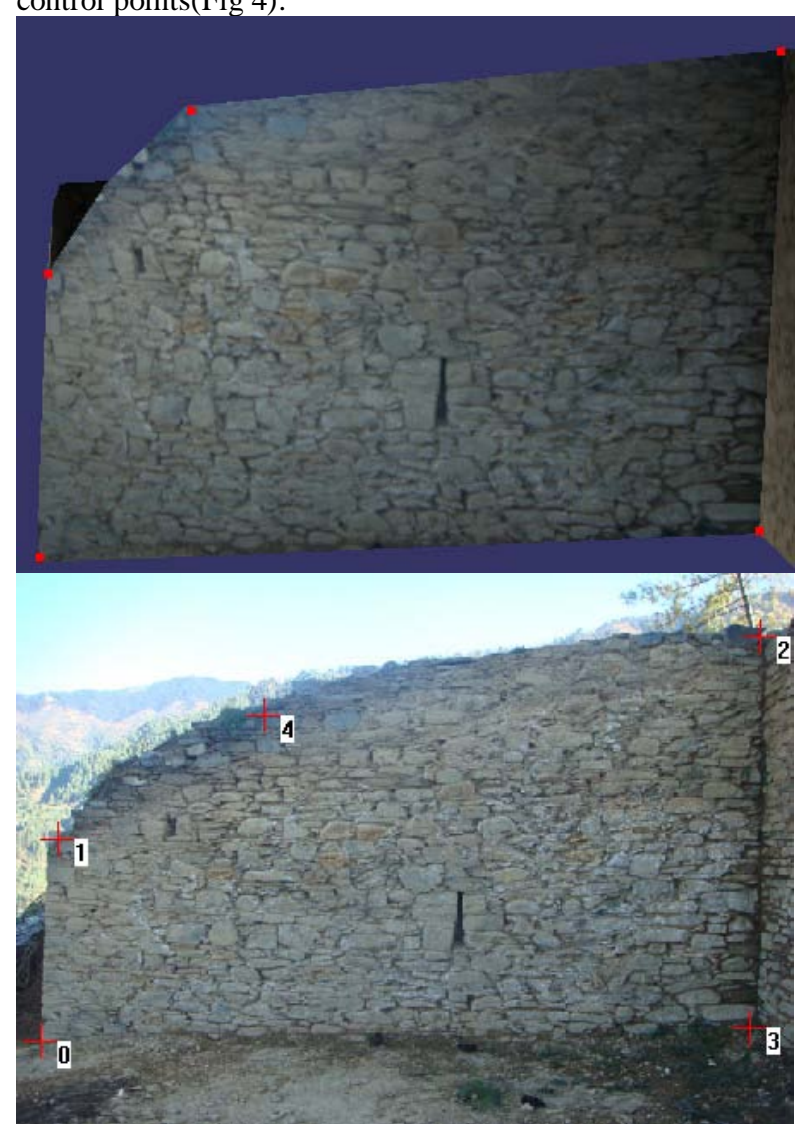

Figure 4: control points based texture mapping.
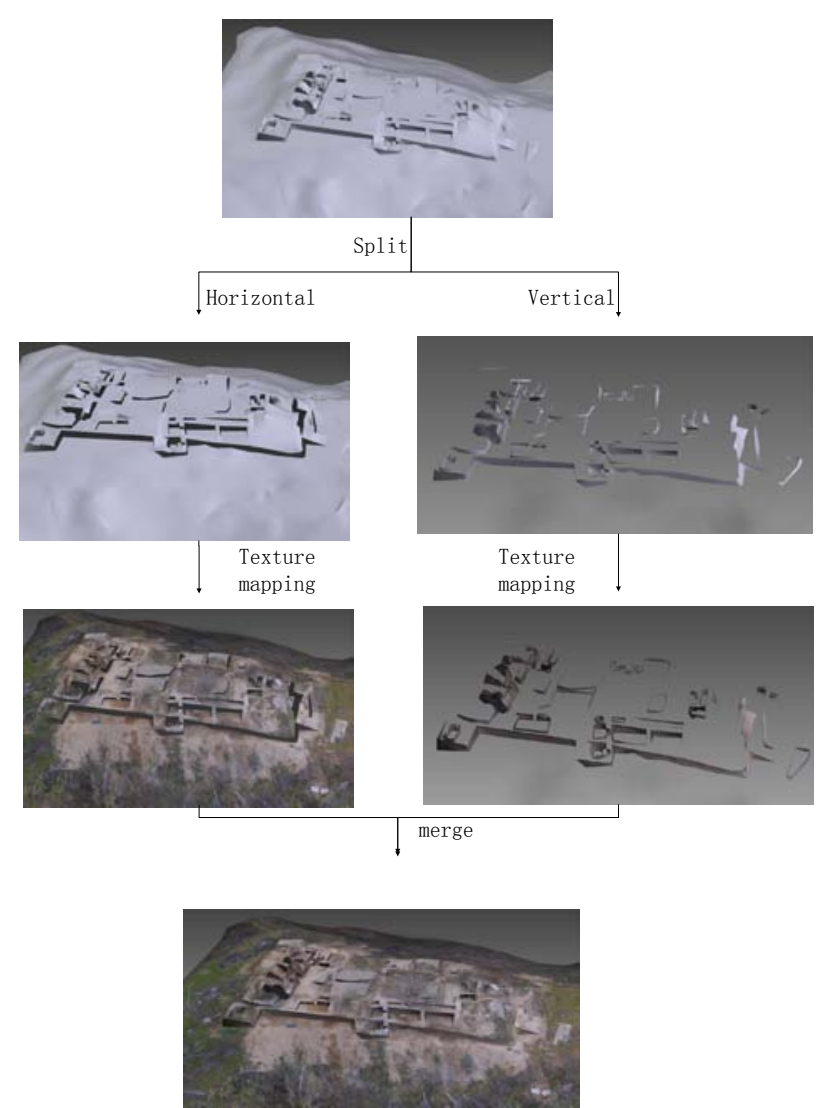

Figure 5: Split and merge texture mapping.

\section{CONCLUSION}

This contribution describes our work for the 3D model generation of Drapham Dzong and its environment using photogrammetric techniques. We have generated textured computer models derived from high-resolution $(0.50 \mathrm{~m}$ pixel size) satellite images, very high-resolution UAV (quadrocopter) aerial images and also from terrestrial images. In many projects, in order to work efficiently and to produce superb results, it is mandatory to use this multi-sensor approach. Another key to success is the close-cooperation between experts in archaeology / cultural heritage and geomatics. There are many pitfalls in data acquisition and processing which can only be avoided with the help of a geomatics expert. On the other hand, when generating the models from images it is indispensable to have indepth knowledge in archaeology, architecture, art history, etc. Otherwise the interpretation of the images by an amateur will inevitably lead to wrong results. According to our experience, UAVs are highly suitable and effective for this kind of work. They combine high flexibility in data acquisition and fast operation at relatively low costs. It can be expected that this technology will be applied a lot more in the future, particularly pertaining to archaeology and cultural heritage applications. Operating such a device in a remote location at $3,000 \mathrm{~m}$ altitude is a challenge to this technology. We experienced a few problems in the field, but they were not serious enough to jeopardize our mission. It can safely be predicted that these problems will be overcome by the system manufacturers in the near future.

In order to achieve optimal results different photogrammetric methods and software packages were applied during the data processing. The final results are an orthoimage of the main castle complex and a digital 3D model of further ruin objects adjacent to the main citadel. These products can be used for further projects e.g. in archeology. In particular, the orthoimage is appropriate for the generation of a site map. Furthermore it is possible to continue processing the present results. Especially some parts of the digital 3D model but also the orthoimage feature shortcomings which can be rectified. Finally, an evaluation of two flights was completed in which two different UAV systems were compared to each other.

\section{ACKNOWLEDGEMENTS}

We would like to acknowledge the contributions of Dr. Fabio Remondino and his group, Bruno Kessler Foundation, Trento, Italy, for generating the Drapham Dzong model. We are also very grateful for the encouragement and permanent understanding and support given by Dr. Eberhard Fischer, SLSA. Without him, the survey would never have been conducted. Furthermore, we would like to acknowledge the contributions of Nagtsho Dorji, Division for Conservation of Architectural Heritage Bhutan, and Walter Roder and Namgyel Tshering from Helvetas Bhutan.

\section{REFERENCE}

CyberCity 3D 2010: CyberCity 3D, Retrieved May 4, 2010, from http://www.cybercity3d.com/.

David, B., Thomas, J. (Eds.) 2008: Handbook of Landscape Archaeology. Walnut Creek, California. 
Dorji, Nagtsho, Fischer, E., Tshering, Namgyel, 2009: The BhutanSwiss Archaeology Project: The first excavation campaign at Drapham Dzong in Central Bhutan; administrative report.

Eisenbeiss, H. 2009: UAV photogrammetry. DISS. ETH NO. 18515, doi:10.3929/ethz-a-005939264.

Fux, P., Sauerbier, M., Kersten, T., Lindstaedt, M., Eisenbeiss, H., 2009: Perspectives and Contrasts: Documentation and interpretation of the petroglyphs of Chichictara, using terrestrial laser scanning and image-based 3D modeling. In: Reindel, M., Wagner, G. (Eds.): New technologies for archaeology. Multidisciplinary investigations in Palpa and Nasca, Peru. Berlin,Heidelberg.

Gruen, A. 2009a: Image-based 3D recording and modeling of landscapes and large cultural heritage sites. In: ARCHAIA - Case Studies on Research Planning, Characterization, Conservation and Management of Archaeological Sites (editors: Nicolo Marchetti and Ingolf

Gruen, A. 2009b: Virtual Archaeology - new methods of imagebased 3D modeling. In: New Technologies for Archaeology (editors: M. Reindel and G.A. Wagner), Natural Science in Archaeology, Springer, Berlin-Heidelberg 2009, pp. 287-305.

iWitness, 2010: "iWitness Close Range Photogrammetry." Retrieved May 1, 2012, from http://www.iwitnessphoto.com/.

Leica-Geosystems 2010: "Leica-Geosystems." Retrieved May 4, 2010, from http://www.leicageosystems.ch.

Meyer, W., 2009: Bhutan-Swiss Archaeology Project Drapham Dzong 08: Results of the first excavation campaign (autumn 2008).

microdrones 2010: "microdrones." Retrieved May 4 2010, from http://www.microdrones.com/.

Photomodeler 2010: "Photomodeler." Retrieved May 5, 2010, from http://www.photomodeler.com/index.htm.

Remondino, F., Gruen, A., von Schwerin, J., Eisenbeiss, H., Rizzi, A., Sauerbier, M., Richards-Rissetto, H., 2009. Multisensor 3D documentation of the Maya site of Copan, Proceedings of the XXIIth CIPA International Symposium, Kyoto, Japan, October 11-15.

Sauerbier, M., Eisenbeiss, H., 2010: UAVs for the documentation of archaeological excavations. IAPRS\&SIS, Vol. 38(5), Newcastle upon Tyne, UK

Thuesen. Archaeopress, Oxford, BAR International Series S1877, 2008, pp. 50-52.

Tobler, W., 1993: Non-isotropic modeling. In: Technical report 93-1, National Center for Geographic Information Analysis. Santa Barbara.

Zhang, L., 2005: Automatic Digital Surface Model (DSM) generation from linear array images. Dissertation ETH No. 16078. IGP Mitteilungen No. 91. Institute of Geodesy and Photogrammetry, ETH Zurich. 